\title{
INFORME DE ACTIVIDADES 2008
}

CENTRO DE DERECHOS HUMANOS

Facultad de Derecho

Universidad de Chile

\section{PALABRAS DE LOS DIRECTORES}
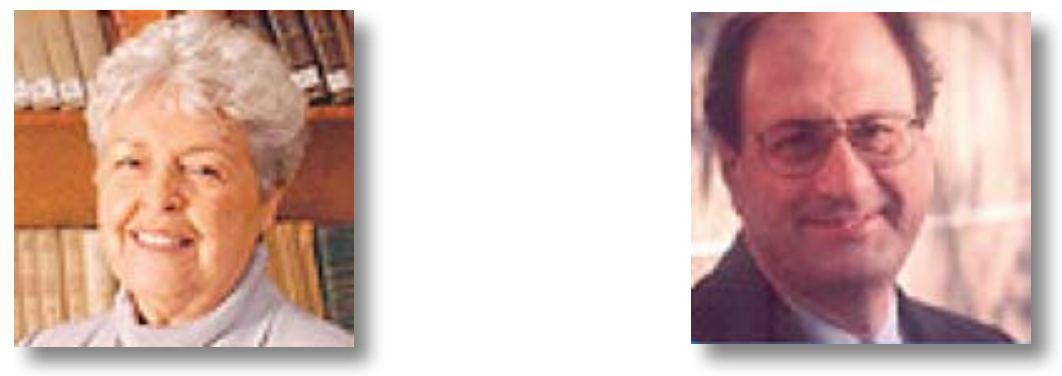

Nos complace presentar el Informe de Actividades 2008 del Centro de Derechos Humanos de la Facultad de Derecho de la Universidad de Chile (CDH).

Durante este año hemos continuado reforzando nuestra labor de docencia, investigación y extensión, y profundizado la labor de cada uno de los Programas que conforman nuestro Centro: Derechos Humanos y Democracia; Mujeres y Derechos Humanos y Derechos Humanos y Estado de Derecho. Cada una de estas áreas ha ejecutado una serie de actividades, contribuyendo al objetivo de hacer de nuestra institución un órgano académico de excelencia, al servicio del avance de los derechos humanos en América Latina.

Una de las actividades principales de nuestro Centro son sus cursos de capacitación para profesionales de países de la región, los que combinan una metodología a distancia y clases presenciales, desarrolladas tanto en Chile como in situ en el extranjero. Estos Diplomados han contado con una gran acogida y se han consolidado como una oportunidad de formación de alto nivel. Asimismo, este año el $\mathrm{CDH}$ ha fortalecido su trabajo en redes a través de reuniones internacionales en materia de derechos de las mujeres y de anticorrupción, las que han permitido reflexionar conjuntamente sobre prioridades y estrategias para el trabajo futuro. Por último, el 2008 se ha caracterizado por una consolidación de nuestras líneas de investigación y extensión, a través de publicaciones y el desarrollo de cursos de especialización dirigidos a la formación de nuevas generaciones en derechos humanos.

Agradecemos a las autoridades de la Universidad de Chile, a nuestros donantes, a los miembros de nuestros consejos consultivos y a nuestro valioso equipo por hacer posible estos logros y por el compromiso con nuestra misión.

CECILIA MEDINA QUIROGA

Co-Directora

Centro de Derechos Humanos
JOSÉ ZALAQUETT DAHER

\author{
Co-Director
}

Centro de Derechos Humanos 


\section{RESUMEN EJECUTIVO}

El Centro de Derechos Humanos de la Facultad de Derecho de la Universidad pone a disposición del público su Informe de Actividades 2008, en el que se dan a conocer las actividades, publicaciones y cifras generales de la institución.

El año 2008 fue clave en la consolidación de las áreas temáticas que aborda el CDH a través de sus tres Programas: Derechos Humanos y Democracia, Mujeres y Derechos Humanos y Derechos Humanos y Estado de Derecho.

El Programa de Democracia se enfocó, principalmente, en el área de Transparencia, Accountability y Lucha contra la Corrupción, ejecutando un Diploma de Postítulo regional sobre este tema y un curso sobre Probidad Pública y Transparencia en Policía de Investigaciones de Chile. En cuanto a su trabajo de extensión, se realizó una Reunión de Especialistas sobre "Transparencia, Rendición de Cuentas y Lucha contra la Corrupción en América Latina: actualizando los desafíos", la cual contó con la participación de destacados académicos y profesionales del área. Por último, se creó un Comité Consultivo del Programa, que sirve de orientación y guía respecto a estrategias y prioridades de acción.

El Programa de Mujeres y Derechos Humanos realizó dos Diplomas de Postítulo. El primero, "Derechos Humanos y Mujeres" tuvo lugar en Santiago de Chile, con una modalidad semipresencial de enseñanza. El segundo, que se realizó en México, fue una nueva experiencia en la actividad docente del $\mathrm{CDH}$, adaptando los contenidos de su Programa a la realidad de dicho país. En cuanto al trabajo en redes, la principal actividad fue la "Iniciativa Latinoamericana para el avance de los Derechos Humanos de las Mujeres", que reunió a destacadas profesionales a reflexionar sobre el el estado actual y estrategias futuras respecto de los derechos humanos de las mujeres en la región. También se desarrolló un Directorio de Instituciones de Derechos Humanos de las Mujeres para Latinoamérica.

El Programa Estado de Derecho se abocó a la capacitación de operadores de justicia a través de su Diploma de Postítulo "Derechos Humanos y Juicio Justo", cuya semana presencial tuvo lugar en Buenos Aires y contó con participantes de varios países latinoamericanos y destacados académicos. También se realizó un Curso para el fortalecimiento de las capacidades de la justicia en la protección de los derechos humanos, en el marco de las sesiones de la Corte Interamericana de Derechos Humanos en Montevideo, Uruguay. En cuanto al trabajo en redes, este Programa actualmente coordina la Red Interamericana de Formación en Derechos Humanos (COLAM) y participa en la Red Latinoamericana y Europea de Derechos Humanos (LAEHR).

Además de las actividades realizadas por los Programas especializados, el 2008 el CDH puso énfasis a su línea de investigación a través de la publicación de diversos libros y artículos. Las principales publicaciones fueron: "Derechos Humanos Hoy. Balance Internacional", libro realizado en el marco de la conmemoración del sexagésimo aniversario de la Declaración Universal de los Derechos Humanos; el Anuario de Derechos Humanos 2008; el libro "Transparencia y Probidad Pública: Estudios de caso en América Latina"; el Compendio de Lecturas sobre Transparencia, Accountability y Lucha contra la Corrupción, difundido por medio de un CD; la actualización del libro sobre "Reparaciones ante la Corte Interamericana de Derechos Humanos". Además, se realizaron numerosas traducciones de artículos, con énfasis en la línea de violencia contra la mujer y estudios de género, así como análisis de jurisprudencia que fueron publicados en el sitio web de cada uno de los Programas. Estos sitios fueron actualizados durante el 2008 y cuentan con completos centros de documentación enfocados a cada una de las áreas prioritarias de capacitación, docencia e investigación del $\mathrm{CDH}$. 
Por último, el CDH realizó importantes actividades académicas a nivel de pre y post grado en la Universidad de Chile, las que son prioritarias para la formación de nuevos académicos y activistas en derechos humanos. Entre las actividades de pregrado se cuentan: el Curso Optativo sobre Derecho Internacional de los Derechos Humanos, dictado tanto en ambos semestres de 2008; un taller de Litigación Internacional en el Sistema Interamericano de Derechos Humanos"; un Concurso de Ensayos en Derechos Humanos. A nivel de Postgrado, se impartió el curso "Derechos Fundamentales. Una perspectiva comparada"; el seminario de magíster "Chile y el Consejo de Derechos Humanos de Naciones Unidas: Desafíos Insospechados"; también se dictaron clases en el Magíster en Derecho con mención en Derecho Público en Talca. Docentes del Centro dirigieron tesis de pregrado y de postgrado.

El detalle de las actividades del Centro de Derechos Humanos se encuentra en el presente Informe Anual de Actividades, que procura difundir y transparentar la labor realizada anualmente por nuestra institución. 


\section{TABLA DE CONTENIDOS}

\section{Misión y Visión}

\section{Programas}

Derechos Humanos y Democracia

Mujeres y Derechos Humanos

Derechos Humanos y Estado de Derecho

\section{Otras Actividades Académicas}

Pregrado

Postgrado

Docencia externa

\section{Extensión}

Sitios Web y Centros de Documentación

Pasantías y visitas

Eventos y conferencias

\section{Publicaciones}

Libros

Publicaciones Online

Publicación del Libro "Transparencia y Probidad Pública. Estudios de caso en América Latina" y del Syllabus CD con un compendio de lecturas

\section{Nuestro Equipo}

Staff del Centro de Derechos Humanos

Consejo Consultivo Nacional y Comité Asesor Internacional

Reconocimientos y premios a nuestro staff

\section{Información Institucional}

Estadísticas

Evaluación de impacto

Instituciones donantes 2008 


\section{MISIÓN Y VISIÓN}

El Centro de Derechos Humanos es una institución académica de alcance regional vinculada a la Facultad de Derecho de la Universidad de Chile. Su misión es promover los derechos humanos y el fortalecimiento de las democracias en América Latina mediante las siguientes líneas de trabajo:

- Educación y capacitación de excelencia a actores relevantes provenientes del Estado y la sociedad civil.

- Investigación enfocada en las necesidades de la realidad regional en materia de derechos humanos.

- Articulación de redes de instituciones y personas que trabajan en derechos humanos a nivel regional y apoyo a iniciativas locales.

- Coordinación de actividades estratégicas a nivel regional.

El CDH tiene tres programas, enfocados en las siguientes áreas temáticas:

\section{Derechos Humanos y Democracia:}

- Procesos de Democratización: Transición a la democracia y superación de un legado de violaciones de derechos humanos y fortalecimiento de la democracia.

- Transparencia, accountability y la lucha contra la corrupción.

\section{Mujeres y Derechos Humanos:}

- Exclusión y discriminación en las realidades latinoamericanas.

- Sistema interamericano de derechos humanos y género.

\section{Derechos Humanos y Estado de Derecho:}

Sistemas internacionales de protección de derechos humanos.

Capacidades internas de los Estados en derechos humanos.

La piedra angular del trabajo del CDH es la capacitación de profesionales mediante Diplomas de Postítulo en derechos humanos. Cada Diplomado aborda un área temática específica y su meta es entregar las herramientas necesarias para avanzar en la agenda de derechos humanos de los países de la región. El Centro apoya el trabajo de la comunidad de egresado/as en sus países de origen, a través de los sitios Web de cada Programa, inaugurados durante el año 2008. La actividad docente de pregrado también es parte central del trabajo del $\mathrm{CDH}$, dentro de la Facultad de Derecho de la Universidad de Chile.

Con el fin de contribuir al progreso, enseñanza y difusión de la disciplina de los derechos humanos, el CDH realiza proyectos de investigación que se han traducido en numerosas publicaciones que pueden descargarse libremente en nuestros sitios Web. Desde el año 2005, el CDH publica cada año el Anuario de Derechos Humanos, libro que sirve como guía y referencia respecto de desarrollos nacionales regionales e internacionales en materia de derechos humanos y campos afines. Otro aspecto del trabajo del CDH está enfocado en el análisis y la planificación estratégica en problemáticas de derechos humanos en América Latina, especialmente en las áreas de transparencia y anticorrupción, y de mujeres y derechos humanos. 


\section{PROGRAMAS}

\section{Derechos Humanos y Democracia}

El Programa de Democracia y Derechos Humanos comprende dos áreas principales de capacitación, investigación y promoción de redes regionales de debate sobre estrategias y prioridades de acción. La primera se ocupa de los Procesos de Democratización en la región, lo que comprende: (i) los principios éticos y legales, así como los condicionamientos políticos y el análisis comparado de experiencias relativos a los procesos de refundación o reconstrucción de la democracia, luego de un período de dictadura, conflictos armados internos $u$ otras situaciones que han dejado un legado de graves violaciones de los derechos humanos; (ii) el fortalecimiento de los sistemas democráticos, incluyendo las instituciones de gobierno, la participación política y la superación de la marginación, discriminación y exclusión social.

La segunda área abarca el campo de la Transparencia, Accountability y Lucha contra la Corrupción. Si bien éste se enmarca dentro de los procesos de fortalecimiento de la democracia, merece una atención especial dado el impacto negativo de la corrupción sobre la gobernabilidad democrática y el ejercicio de derechos, así como por el hecho de que en torno a él se ha desarrollado un campo específico (todavía emergente) de ética política, políticas públicas y acción ciudadana.

\section{a. Capacitación}

\section{i. Diploma de Postítulo en Transparencia, Accountability y Lucha contra la Corrupción}

La primera versión del Diploma de Postítulo en Transparencia, Accountability y Lucha contra la Corrupción (DIPCOR) fue organizada por el Programa de Democracia con el apoyo de Foundation Open Society Institute. Se llevó a cabo entre marzo y julio del 2008 y ofreció a 25 profesionales provenientes de diversos países de América Latina la posibilidad de adquirir una importante base teórica sobre los conceptos fundamentales en este campo; el estudio de los principales ámbitos y problemas de corrupción; y, por último, la revisión de estrategias para la construcción de sociedades transparentes, justas, participativas y respetuosas de los derechos humanos.

Este Diplomado pretende capacitar a un grupo de profesionales de diversas disciplinas, con el objeto de intercambiar conocimientos y experiencias que permitan a cada uno colaborar -sea desde el sector público, privado o no gubernamental- con el fortalecimiento de la democracia y la integridad pública en sus países.

El curso combinó 17 semanas de enseñanza en línea con tres semanas de clases presenciales en Santiago de Chile. Además del personal docente del $\mathrm{CDH}$, participaron en el transcurso de estas semanas prestigiosos profesionales del ámbito académico y público. Sus clases ofrecieron la oportunidad de debatir los desafíos que enfrentan los procesos de fortalecimiento democrático y modernización del Estado, la influencia desmedida de los agentes privados en la política y la regulación del lobby, las diversas agendas de probidad, la relación del fenómeno de la corrupción con el poder judicial y la justicia transicional, el derecho de acceso a la información pública, la pobreza y clientelismo en los programas sociales, entre otros temas.

ii. Curso sobre Probidad Pública y Transparencia en Policía de Investigaciones de Chile

En el marco del convenio entre la Facultad de Derecho de la Universidad de Chile y Policía de Investigaciones de Chile, y a pedido de esta última, el CDH impartió dos cursos breves sobre probidad pública, transparencia, anticorrupción y la nueva ley de Acceso a Información Pública, 
a miembros de dicha entidad. El primero de estos cursos estuvo dirigido a cerca de 50 personas de rango medio y alto dentro de la institución, mientras que el segundo, que contó con la participación de 25 personas, estuvo dirigido a altos mandos, incluyendo jefes regionales. Ambos cursos fueron dictados por el Co-Director del $\mathrm{CDH}$, profesor José Zalaquett.

\section{b. Investigación}

i. Publicación del Libro "Transparencia y Probidad Pública. Estudios de caso en América Latina"

En abril de 2008, el Programa de Democracia y Derechos Humanos, con el apoyo de Foundation Open Society Institute, presentó el libro "Transparencia y Probidad Pública. Estudios de caso en América Latina", editado por José Zalaquett y Alex Muñoz. Esta obra reúne 13 artículos y estudios de caso de experiencias y situaciones relativas a transparencia, accountability y lucha contra la corrupción en América Latina, con el fin de servir para la capacitación para profesionales en esta área.

ii. Transparencia, Accountability y Lucha contra la Corrupción: Syllabus CD.Compendio de lecturas 2008

En agosto de 2008, el Programa puso a disposición del público, gracias al apoyo de Open Society Institute, un nuevo CD que reúne 47 artículos sobre casos y tendencias actuales en materia de transparencia y lucha contra la corrupción.

En esta publicación figuran textos de diversos autores internacionales -expertos, académicos y juristas- que forman parte del material de lectura (syllabus) del Diploma de Postítulo sobre Transparencia, Accountability y Lucha contra la Corrupción.

El CD está disponible en el sitio web del $\mathrm{CDH}$ y en la página del área de transparencia $\boldsymbol{w} \boldsymbol{w} \boldsymbol{w}$. transparenciacdh.uchile.cl

\section{c. Trabajo en Redes}

\section{i. Creación de un Comité Consultivo}

Desde fines de julio de 2008, el área de Transparencia, Accountability y Lucha contra la Corrupción cuenta con un Comité Consultivo internacional conformado por destacados académicos y profesionales. Este Comité cumple funciones de orientación y guía respecto a estrategias y prioridades de acción, además de participación en reuniones y clases. La lista de sus integrantes consta en el sitio web del Programa.

ii. Segunda Reunión Regional de Especialistas "Transparencia, Rendición de Cuentas y Lucha Contra la Corrupción en América Latina: actualizando los desafíos"

Entre el 24 y 26 de noviembre de 2008 se realizó la Segunda Reunión Regional sobre Transparencia, Rendición de Cuentas y Lucha Contra la Corrupción en América Latina. Esta actividad contó con el apoyo de la Fundación Tinker y la participación de 16 especialistas provenientes de diez países de la región, Europa y Estados Unidos.

Algunas de las prioridades y estrategias identificadas durante este encuentro fueron: explorar las conexiones entre los derechos humanos y la lucha contra la corrupción, tanto a nivel teórico como en la práctica; avanzar en el desarrollo del acceso a la información en la región, con el desarrollo 
de archivos y la promoción de demanda por información; y revisar el impacto de la corrupción en la marginación y exclusión de grupos vulnerables.

El programa prepara para el 2009 una publicación con el Informe final de esta reunión.

\section{iii. Visitas a Brasil y Venezuela}

Con el fin de difundir el Programa e incrementar el contacto con instituciones académicas y de la sociedad civil brasileña y venezolana, se realizaron viajes a Brasilia, San Pablo y Caracas, donde se sostuvieron reuniones con representantes de diversas organizaciones, universidades e instituciones públicas.

\section{Mujeres y Derechos Humanos}

El Programa de Mujeres y Derechos Humanos está dedicado a la investigación y docencia en el área de los derechos humanos de las mujeres. El Programa también participa en el desarrollo de redes académicas y de organizaciones de la sociedad civil. A través de su página web y sus publicaciones apoya el trabajo de sus ex alumnas/os y de quienes defienden los derechos de las mujeres en la región.

El Programa pone énfasis en el análisis de los fenómenos de desigualdad y exclusión que enfrentan las mujeres de la región, utilizando como marcos teóricos el derecho internacional y las distintas corrientes de la teoría feminista. Con ese enfoque, se estudia el impacto de la discriminación en el acceso y goce a los derechos humanos, especialmente los derechos políticos, el derecho a vivir una vida libre de violencia, la autonomía económica y los derechos sexuales y reproductivos.

\section{a. Capacitación}

\section{i. Diploma de Postítulo en "Derechos Humanos y Mujeres: Teoría y Práctica"}

Este diploma de postítulo, dictado desde 2004, tiene como principal objetivo capacitar a abogadas/os con experiencia en el ámbito de los derechos de las mujeres, para que puedan aplicar, tanto en el plano nacional como internacional, el derecho internacional de los derechos humanos y sus mecanismos de protección, a favor de mujeres y niñas cuyos derechos son vulnerados. El curso incorpora la teoría de género aplicada al derecho con un enfoque práctico, permitiendo a las/los alumnas/os trabajar en la superación de la subordinación y de la desigualdad que afectan a las mujeres en las áreas de acceso al poder, violencia, autonomía económica y derechos sexuales y reproductivos.

Desde el 4 de agosto al 19 de diciembre de 2008, 25 estudiantes provenientes de diversos países de América Latina cursaron este Diploma, el cual combinó tres semanas de clases presenciales y 15 semanas de clases en línea. Además de los docentes del $\mathrm{CDH}$, dictaron clases y conferencias destacados/as académicos - nacionales y extranjeros- expertos en la materia.

Durante la semana presencial, realizada en diciembre, tuvo lugar el encuentro "Gobierno paritario y su impacto en el espacio público", organizado conjuntamente por el CDH y Corporación Humanas. El coloquio contó con la presencia de ministras y ex ministras del gobierno de la Presidenta Michelle Bachelet.

\section{ii. Diploma de Postítulo in situ "Derechos Humanos y Mujeres: México"}

Durante el primer semestre de 2008, México fue sede del primer curso in situ impartido por el Programa Mujeres y Derechos Humanos, el cual se realizó conjuntamente con la Oficina del 
Alto Comisionado de Naciones Unidas para los Derechos Humanos, oficina México, el Instituto Tecnológico Autónomo de México (ITAM) y UNIFEM.

Este curso estuvo dirigido a 23 abogadas y abogados que se dedicarán al trabajo por la defensa y protección de los derechos de las mujeres, y replicó, en lo sustancial, el diploma de postítulo "Derechos Humanos y Mujeres: Teoría y Práctica".

El diseño y búsqueda de los temas y materiales de este Diploma de Postítulo fue especialmente adaptado a la realidad mexicana, como resultado de un proceso en el cual participaron académicas/os de Chile y México.

Durante el primer semestre del año 2009 tendrá lugar la segunda versión de los Diplomas de Postítulo in situ, en Bogotá, Colombia.

\section{b. Investigación}

i. Traducción de artículo "The Path to Gender Justice in the Inter-American Court of Human Rights" (El Camino hacia la Justicia de Género en la Corte Interamericana de Derechos Humanos)

Durante el 2008, el CDH tradujo el artículo de la investigadora Patricia Palacios Zuloaga "The Path to Gender Justice in the Inter-American Court of Human Rights", para su uso en el diploma de postítulo "Derechos Humanos y Mujeres: Teoría y Práctica". Este artículo ganó el Premio otorgado por The Bernard and Audre Rapoport Center for Human Rights and Justice, de la Universidad de Texas, para los Derechos Humanos de las Mujeres, y fue publicado en el Texas Journal of Women and the Law.

ii. Tratamiento de la violencia contra las mujeres en el sistema de Naciones Unidas

Durante el primer semestre de 2008, Patricia Palacios Zuloaga desarrolló una investigación sobre el tratamiento de la violencia contra las mujeres en el sistema universal de protección de los derechos humanos.

\section{c. Trabajo en Redes}

i. "Iniciativa Latinoamericana para el avance de los Derechos Humanos de las Mujeres"

El objetivo de este proyecto fue realizar un diagnóstico del estado de avance de los derechos humanos de las mujeres en la región, analizar las estrategias desarrolladas con miras a mejorar iniciativas futuras e identificar temas prioritarios de trabajo e investigación para los próximos años.

Las principales actividades fueron:

- Formar un grupo de destacadas expertas en el ámbito de la investigación, el derecho y las políticas públicas, quienes fueron convocadas por su trayectoria como activistas del movimiento de mujeres.

- Consultar previamente a las convocadas, mediante cuestionarios y entrevistas.

- Sobre esa base, elaborar un documento preparatorio que sistematizó los principales puntos de vista expresados en los cuestionarios y entrevistas.

- Realizar una reunión regional de expertas, desarrollada en la Facultad de Derecho de la Universidad de Chile los días 18, 19 y 20 de junio de 2008. 
Durante la reunión, las participantes presentaron estrategias significativas desarrolladas en sus países, con el fin de ponderar avances y logros alcanzados, e identificar barreras y oportunidades en el avance de los derechos humanos de las mujeres.

A partir del proceso de preparación y de la reunión, se elaboró un documento de evaluación que recogió las opiniones expresadas por las participantes respecto al diseño de la reunión y las posibles formas de dar continuidad a la Iniciativa durante los próximos años. Los resultados de este proyecto serán analizados en un libro, que se publicará el primer semestre de 2009.

\section{ii. Directorio de Instituciones de los Derechos Humanos de las Mujeres Latinoamericanas}

Durante el 2008 se colocó en el sitio www.programamujerescdh.cl, un directorio de instituciones que trabajan en derechos humanos de las mujeres en América Latina. El directorio contiene, entre otras instituciones, entidades gubernamentales e internacionales, órganos de los poderes judicial y legislativo de distintos países, universidades y centros de estudios.

\section{iii. Conferencia Internacional sobre Género y Derecho}

Durante el segundo semestre de 2008, el Programa se adjudicó el proyecto para organizar y realizar una conferencia sobre género y derecho de la Red Latinoamericana de Académico/as del Derecho (Red ALAS).

Este evento, de alcance internacional, se realizará los días 13 y 14 de julio de 2009 en la Facultad de Derecho de la Universidad de Chile.

\section{iv. Asesorías en procesos institucionales}

El Programa Mujeres y Derechos Humanos colaboró en la elaboración del marco del curso "Conocimiento y Manejo de Instrumentos Internacionales para la Defensa de los Derechos Humanos de las Mujeres Indígenas". Participan también en esta iniciativa UNIFEM, la Oficina del Alto Comisionado de Naciones Unidas para los Derechos Humanos, IDEA Internacional, Diakonia y la Universidad Andina.

\section{Programa Estado de Derecho y Derechos Humanos}

El Programa Estado de Derecho busca contribuir al respeto y protección de los derechos humanos por parte de los Estados americanos, a través de actividades de docencia e investigación que apoyen el fortalecimiento de los sistemas de protección nacional e internacional de los derechos humanos. Ello supone que los Estados adecuen sus normas, instituciones y procedimientos nacionales a las obligaciones internacionales que han contraído.

Este Programa se organiza en torno a dos ejes temáticos: (i) Los sistemas internacionales de protección de derechos humanos, con especial énfasis en el sistema interamericano, y (ii) Capacidades internas de los Estados en materia de derechos humanos.

Para la consecución de sus objetivos, el Programa ofrece la capacitación de funcionarios del Estado, particularmente operadores de justicia (jueces, defensores y fiscales) sobre la base de cursos diseñados según las necesidades de cada institución. Asimismo, proporciona apoyo docente a los organismos nacionales y regionales que trabajan en temas afines. El Programa, además, desarrolla líneas de investigación y trabaja por el afianzamiento de redes regionales de formación en derechos humanos. 


\section{a. Capacitación}

\section{i. Diploma de Postítulo sobre "Derechos Humanos y Juicio Justo"}

El Diploma "Derechos Humanos y Juicio Justo" surgió del trabajo conjunto de las diversas instituciones académicas que integran la Red Interamericana de Formación en Derechos Humanos, cuya coordinación se encuentra a cargo del Director del Programa, Dr. Claudio Nash Rojas.

Este curso busca brindar capacitación de excelencia a los profesionales que intervienen en la administración de justicia en América Latina -jueces, defensores públicos y fiscales- en el uso de los estándares interamericanos de derechos humanos, particularmente en materia de debido proceso y discriminación. Se procura que los operadores de justicia puedan replicar los conocimientos adquiridos en sus labores cotidianas. De este modo, se busca armonizar el derecho y jurisprudencia interna con la normativa y jurisprudencia internacional de derechos humanos.

Este Diplomado, desarrollado entre septiembre y noviembre de 2008, tuvo una duración de 12 semanas y contempló una fase de enseñanza a distancia y otra de sesiones presenciales, desarrolladas en Buenos Aires, Argentina. En esta última fase, las personas participantes asistieron a clases magistrales, destinadas al análisis de casos, y a plenarios de discusión, los que contaron con el concurso de académicos de renombre internacional.

Esta iniciativa constituye el primer curso subregional, dentro de un proyecto que comprende la realización de un curso en México (para ese país y para Centroamérica) a realizarse durante el 2009, y uno de carácter regional, para el 2010.

ii. Curso para el fortalecimiento de las capacidades de los operadores de justicia para la protección de los derechos humanos, en Montevideo.

Durante el segundo semestre de 2008, en el marco de un esfuerzo que está desarrollando la Corte Interamericana de Derechos Humanos, con el apoyo de la Fundación Konrad Adenauer, para implementar cursos de formación sobre el sistema interamericano de derechos humanos, se desarrolló en Uruguay un Curso para el Fortalecimiento de las Capacidades de los Operadores de Justicia para la Protección de los Derechos Humanos, dirigido a jueces a nivel nacional.

Durante el período de sesiones de la Corte, realizado en Uruguay, el Programa de Estado de Derecho, a través de su Director, Dr. Claudio Nash, participó en este curso, realizado en conjunto con el CEJU (Centro de Estudios Judiciales del Uruguay) en Montevideo.

\section{b. Investigación}

En programa Estado de Derecho realiza iniciativas de investigación en dos líneas fundamentales: análisis de jurisprudencia y justicia constitucional. Se han confeccionado los siguientes materiales y publicaciones:

\section{i. Material de Estudio para Operadores de Justicia.}

Con el objeto de apoyar y dar bases sólidas al Diploma de Postítulo "Derechos Humanos y Juicio Justo", se confeccionó un material de estudio con textos especialmente elaborados para esta actividad académica. El material fue puesto a disposición de los participantes a través de la plataforma web del curso. 
ii. Segunda Edición, revisada y actualizada del libro "Reparaciones ante la Corte Interamericana de Derechos Humanos"

En el segundo semestre de 2008, se elaboró una edición revisada y actualizada del libro "Reparaciones ante la Corte Interamericana de Derechos Humanos".

\section{iii. Estudio de Jurisprudencia de la Corte Europea de Derechos Humanos}

Esta investigación, desarrollada durante el primer semestre de 2008 y llevada a cabo en conjunto con el pasante Gabriel Querry, de la Universidad de Ottawa, tuvo por objeto la revisión de los estándares desarrollados por el sistema europeo de protección de los derechos humanos en materia de debido proceso.

\section{iv. Publicación de artículos sobre justicia constitucional.}

Con el objeto de contribuir a la profundización y desarrollo del debate sobre temas de justicia constitucional, el Director del Programa Estado de Derecho, elaboró los siguientes artículos académicos, los cuales se encuentran disponibles en el sitio web del Programa: "Los derechos fundamentales: el desafío para el constitucionalismo chileno del siglo XXI"; "Los derechos fundamentales: debates actuales y nuevos desafíos" y "La aplicación judicial de los tratados internacionales". Este último en conjunto con Alberto León Gómez, Loretta Ortiz y César Landa.

\section{c. Trabajo en Redes}

\section{i. Coordinación de la Red Interamericana de Formación en Derechos Humanos (COLAM)}

Desde el año 2007, el Programa de Estado de Derecho, a través de su Director, coordina la Red Interamericana de Formación en Derechos Humanos . Ésta es una red de derechos humanos de carácter interuniversitario destinada a apoyar la formación de jueces, fiscales y defensores públicos en el uso de los estándares interamericanos de derechos humanos.

La Red fue creada por el Colegio de las Américas de Canadá (COLAM), programa de la OUI (Organización Universitaria Interamericana) cuyo principal objetivo es fomentar la cooperación entre las universidades del continente a través de la creación de redes interamericanas de formación e investigación.

\section{ii. Participación en $L A E H R$}

La Red Latinoamericana y Europea de Derechos Humanos (LAEHR), creada en el año 2005 gracias al financiamiento otorgado por el Programa Alfa, agrupa y coordina a universidades y ONG's de diversos países latinoamericanos y europeos. Su principal objetivo es fortalecer las capacidades de enseñanza en el campo de los derechos humanos, tanto en Latinoamérica como en Europa, con especial énfasis en el ámbito de la investigación.

Entre los miembros de esta Red se encuentra el $\mathrm{CDH}$, el que por medio de su Programa Estado de Derecho, participa en la reunión anual que organiza LAEHR y ha presentado dos proyectos vinculados a la docencia en el ámbito del derecho internacional de derechos humanos. 


\section{OTRAS ACTIVIDADES ACADÉMICAS}

\section{Pregrado}

La actividad docente a nivel de pregrado forma parte central del trabajo del CDH en la Facultad de Derecho de la Universidad de Chile, ya que éste es el terreno de formación de nuevos académicos y activistas en derechos humanos tanto para Chile como para la región. En este marco, el $\mathrm{CDH}$ dicta diversos cursos y realiza una serie de otras actividades académicas dirigidas a los estudiantes de pregrado.

\section{a. Curso "Derecho Internacional de los Derechos Humanos"}

El propósito de este curso es que los alumnos comprendan la gestación, naturaleza y desarrollo del Derecho Internacional de los Derechos Humanos y su relación con otros sistemas humanitarios; que conozcan los principales componentes normativos de dichos sistemas, sus sistemas jurídicos de protección y sus principales instituciones; y, por último, que adviertan las posibilidades de actuar en estos campos como ciudadanos y profesionales.

El primer semestre de 2008 el curso fue impartido por el Director del Programa Estado de Derecho, Claudio Nash, y el segundo semestre, por el Co-Director del CDH, José Zalaquett.

El curso se dicta desde 2002 y anualmente lo aprueban cerca de 180 alumnas/os.

\section{b. Taller "Litigación Internacional en el Sistema Interamericano de Derechos Humanos" y par- ticipación en concursos internacionales}

Como parte del proceso de formación y capacitación en materia de derechos humanos, el CDH prepara anualmente a estudiantes de pregrado para participar en el Concurso Interamericano de Derechos Humanos, organizado por la American University. Dicho concurso es una instancia de educación y discusión sobre el sistema interamericano en el cual participan más de 50 universidades de Latinoamérica, el Caribe y Norteamérica.

Durante el segundo semestre de 2008, la preparación para el certamen se realizó a través de la implementación de un curso profundizado sobre "Litigación Internacional en el Sistema Interamericano de Derechos Humanos", inédito en el país. El Taller tuvo una primera etapa de clases presenciales, seguida de talleres prácticos y clases de expresión oral y corporal impartidas por la especialista Claudia Berguer. El curso estuvo a cargo de la Co-Directora del CDH, Cecilia Medina y del profesor Claudio Nash. La coordinación estuvo a cargo de Alia Trabucco Zerán, asistente de dirección del $\mathrm{CDH}$.

El Taller culminó con la simulación de un juicio oral ante la Corte Interamericana, en el cual se seleccionó a Tomás Pascual y Gustavo Namuncura como la pareja que representará a la Facultad de Derecho en el XIV Concurso Interamericano de Derechos Humanos de la American University.

\section{c. Memorias de pregrado}

Otro de los ejes centrales del CDH es el fomento de la investigación. En ese entendido, se busca promover y colaborar en la ejecución de memorias y tesis sobre temas relevantes vinculados a las materias de interés para el Centro.

Durante el año el profesor Claudio Nash dirigió la tesis de pregrado de Bernardita Francisca Díaz Gude y Claudia Paz Escobar Díaz, Ilamada "La Violación como Crimen de Guerra. De Ginebra a Roma". 
El Co-director José Zalaquett dirigió la memoria de Pilar Iturrieta y Francisco José Toyos "Migraciones Internacionales y Derechos Humanos".

La Co-directora Cecilia Medina guió la memoria de licenciatura "Dos concepciones de igualdad en el sistema interamericano. Una superposición que amenaza a las acciones afirmativas" de Valeska David Contreras y Alia Trabucco Zerán.

\section{d. Primer Concurso de Ensayos en Derechos Humanos}

En el marco de SUS actividades académicas y de extensión, el CDH realizó su primera convocatoria a un Concurso de Ensayos en Derechos Humanos, realizado en el marco del Primer Congreso de Derechos Humanos, organizado por alumnos de la Facultad de Derecho de la Universidad de Chile.

El Concurso convocó a estudiantes de pregrado a nivel nacional, recibiéndose un número importante de trabajos. El jurado estuvo conformado por los profesores Cecilia Medina, José Zalaquett y Claudio Nash, quienes determinarán el ensayo ganador a efectos de evaluar su posible publicación en el Anuario de Derechos Humanos 2009.

\section{e. Grupos de ayudantes}

Un elemento clave en el trabajo del $\mathrm{CDH}$ a nivel de pregrado es la formación de nuevos académicos. Para ello, en cada Programa se ha integrado un equipo estudiantes de pregrado que colaboran en labores de investigación y gestión.

\section{Postgrado}

\section{a. Curso de Magíster: "Derechos Fundamentales. Una perspectiva comparada"}

El Director del Programa de Estado de Derecho dictó el curso "Derechos Fundamentales. Una perspectiva comparada" para el Programa de Magíster de la Escuela de Graduados de la Facultad de Derecho de la Universidad de Chile.

\section{b. Seminario Magíster: "Chile y el Consejo de Derechos Humanos de Naciones Unidas. Desafíos Insospechados"}

Durante el segundo semestre del año 2008, el profesor Claudio Nash continuó profundizando en clases de magíster a través de un seminario relativo a los desafíos de Chile ante el Consejo de Derechos Humanos de Naciones Unidas.

\section{c. Magíster en Derecho con mención en Derecho Público en Talca: Impacto del Sistema Internacional de Derechos Humanos en Chile}

La Escuela de Graduados de la Facultad de Derecho desarrolla maestrías en diferentes ciudades. Una de ellas fue el Magíster en Derecho con mención en Derecho Público en Talca. Este contempla una serie de módulos, uno de ellos destinado al tema del impacto del Sistema Internacional de Derechos Humanos en Chile. A cargo de este módulo estuvieron la profesora Cecilia Medina y Dr. Claudio Nash.

\section{d. Comité Académico Doctorado en Derecho}

La Co-Directora Cecilia Medina y el Dr. Claudio Nash son miembros del Comité Académico del Doctorado en Derecho ofrecido por la Universidad de Chile. 


\section{e. Curso Tutorial de Lecturas: Derechos Fundamentales. Tendencias Jurisprudenciales en Latinoamérica. Doctorado en Derecho, Universidad de Chile}

Este curso centró la discusión en torno a la jurisprudencia constitucional respecto de los elementos que permiten definir un concepto de derechos fundamentales. Se discutieron las visiones tradicionales y los aportes desde la jurisprudencia constitucional en ciertos países líderes de la región.

\section{f. Tesis de Postgrado}

El profesor Claudio Nash dirigió la tesis de magíster "Adpic y Adpic Plus: Un Análisis Integral del Derecho Internacional", de Nadiafna Rodríguez P.

La profesora Cecilia Medina guía, como cosupervisora, una tesis de Master para la Universidad de Oxford sobre un análisis feminista de igualdad y discriminación aplicado al sistema interamericano y una tesis de doctorado en el Programa de la Facultad de Derecho de la Universidad de Chile, titulada "Proposición de un régimen jurídico de protección de la vida del que está por nacer compatible con los derechos fundamentales reconocidos en la Constitución Política de Chile y el derecho internacional de los derechos humanos", de Verónica Undurraga. Finalmente, cosupervisa una tesis doctoral sobre desapariciones para la Universidad de Utrecht, Holanda.

\section{g. Consejo Escuela de Graduados y Consejo de Evaluación Universidad de Chile}

El Co-Director José Zalaquett es miembro del Consejo Escuela de Graduados de la Facultad de Derecho y del Consejo de Evaluación de la Universidad de Chile.

\section{Docencia Externa}

Dentro de las actividades permanentes de los/as académicos y Co-Directores del CDH se encuentra la docencia externa. Durante el 2008, los miembros del equipo asistieron a reuniones, congresos e impartieron conferencias en diversos países, incluidos Estados Unidos, Canadá, Holanda, Italia, Grecia, Brasil, Argentina, Uruguay, México, Venezuela, Perú y Noruega.

\section{EXTENSIÓN}

\section{Sitios Web y Centros de Documentación}

El CDH tuvo grandes avances en su presencia en Internet, con el lanzamiento de nuevos sitios especializados para sus Programas y mayor contenido de recursos online.

Los logros más importantes durante el año 2008 fueron la puesta en marcha de estos sitios web y la creación de centros de documentación.

a) Programa Democracia y Derechos Humanos: Su Centro de Documentación contiene una amplia gama de información sobre el tema de Transparencia y combate contra la Corrupción. Es posible acceder a un compendio de normativa y decisiones internacionales sobre la materia; una selección de las normas nacionales relevantes; y, por último, una bibliografía clasificada por temas.

b) Programa de Mujeres y Derechos Humanos: En el Centro de Documentación se abordan, fundamentalmente, los siguientes temas: derecho y jurisprudencia internacional; derecho comparado y jurisprudencia latinoamericana; bibliografía sobre género sistematizada por temas; y artículos de interés sobre estudios de mujeres, género y derecho. 
c) Programa Estado de Derecho: En su Centro de Documentación hay jurisprudencia sobre el sistema universal y regional de derechos humanos, acerca de: hábeas corpus; derecho a defensa; prisión preventiva; presunción de inocencia; tribunales militares; condiciones de detención; desaparición forzada; tortura; discriminación; impunidad; terrorismo; refugiados.

El rediseño, expansión y actualización de los sitios del $\mathrm{CDH}$ ha producido un gran aumento de visitas de prácticamente todos los países de América Latina. En el transcurso de un mes, se registran 75.500 páginas vistas. El número de sesiones de un solo mes es equivalente a la cifra de seis meses del año 2007.

\section{Pasantías y visitas}

El CDH profundiza también su labor de extensión, docencia e investigación a través de estancias de investigación, pasantías y visitas. Durante el 2008 realizaron pasantías Gabriel Querry, alumno de la Universidad de Ottawa; Yoel Bitrán, alumno de la Universidad de Princeton, y Kelly Talon, proveniente de la Universidad de Harvard.

\section{Eventos y conferencias}

El CDH participó como colaborador en la organización y financiamiento del Primer Congreso de Derechos Humanos de la Universidad de Chile, dirigido a estudiantes y académicos de todo el país y que contó con importantes personalidades del mundo de los derechos humanos. Asimismo, colaboró en la organización de una conferencia sobre Derechos Humanos y Medio Ambiente.

Por otro lado, los distintos académicos/as del $\mathrm{CDH}$ y los Co-Directores participaron en numerosas conferencias y actividades de docencia externa, tanto en Chile como en el extranjero.

\section{PUBLICACIONES}

\section{Libros}

El CDH ha realizado varios proyectos de investigación, los que se han traducido en las publicaciones mencionadas en cada uno de los Programas del $\mathrm{CDH}$, adicionalmente, en los siguientes libros:

\section{a. Anuario de Derechos Humanos 2008}

Desde el año 2005 a la fecha, el CDH publica el Anuario de Derechos Humanos de Chile, libro de referencia y consulta en materia de derechos humanos y campos afines. Este texto pone a disposición del público, de manera gratuita, los distintos desarrollos en este campo ocurridos dentro del período anual cubierto por cada edición. Su editora es Claudia Sarmiento.

En su edición 2008, el Anuario abordó temáticas como la prohibición de discriminación, la dimensión moral de los derechos humanos, un simposio sobre igualdad de oportunidades, otros temas de debate nacional e internacional, una entrevista a la académica estadounidense Rhonda Copelon y el tema emergente de comercio internacional y derechos humanos.

\section{b. Derechos Humanos Hoy. Balance Internacional}

El sexagésimo aniversario de la Declaración Universal de los Derechos Humanos fue un hito de trascendencia que el $\mathrm{CDH}$ decidió conmemorar a través de una publicación. Treinta y dos autores y autoras participaron en la creación del libro "Derechos Humanos Hoy. Balance Internacional". 
Éste contempla, en su primera parte, análisis sobre el estado actual y desafíos de los derechos humanos a nivel global y regional a través de ensayos inéditos de Aryeh Neier, Louise Arbour, Chidi Odinkalu, Víctor Abramovich, Hina Jilani, Thomas Hammarberg y Hanny Megally. La segunda parte de la publicación contó con la participación de otros destacados académicos, quienes reflexionaron sobre el Movimiento de Derechos Humanos, la Democracia, Sistemas Regionales de Protección, Derechos Económicos, Sociales y Culturales, Derechos de las Mujeres, Diversidad Sexual, Migraciones, Seguridad Internacional, Justicia Penal Internacional, Pueblos Indígenas, Multiculturalismo y Medio Ambiente. La edición del libro estuvo a cargo de Alia Trabucco Zerán.

Esta publicación es distribuida por Editorial Catalonia, a través de sus tiendas y sitio web www. catalonia.cl.

\section{c. Publicación del Libro "Transparencia y Probidad Pública. Estudios de caso en América Latina" y del Transparencia, Accountability y Lucha contra la Corrupción: Syllabus CD. Compendio de lecturas 2008}

Como fue descrito en el Programa Democracia y Derechos Humanos, durante el 2008 se presentó el libro "Transparencia y Probidad Pública. Estudios de caso de América Latina", editado por José Zalaquett y Alex Muñoz. Asimismo, en agosto se puso a disposición del público, gracias al apoyo de Open Society Institute, un nuevo CD que reúne 47 artículos sobre casos y tendencias actuales en materia de transparencia y lucha contra la corrupción.

Ambos están disponibles en el sitio www.transparenciacdh.uchile.cl.

\section{NUESTRO EQUIPO}

\section{i. Staff del Centro de Derechos Humanos}

\section{Dirección}

- Cecilia Medina Quiroga, Co-Directora.

- José Zalaquett Daher, Co-Director.

- Alia Trabucco Zerán, Asistente de Dirección.

\section{Programa de Mujeres y Derechos Humanos}

- Verónica Undurraga Valdés, Directora del Programa.

- Marcela Sandoval Osorio, Investigadora.

- Claudia Sarmiento Ramírez, Investigadora.

\section{Programa de Estado de Derecho}

- Claudio Nash Rojas, Director del Programa.

- Valeska David Contreras, Asistente de Investigación.

- Ignacio Mujica, Asistente de Investigación. 


\section{Programa de Derechos Humanos y Democracia}

- Silvana Lauzán, Directora del Programa.

- Constanza Toro Justiniano, Investigadora.

- Sebastián Figueroa Rubio, Asistente de Investigación.

\section{5. Área de Administración y Finanzas}

- Nelly Berenguer, Directora del Área.

- Ingrid Wittebroodt, Coordinadora de Diplomas de Postítulo.

- María Victoria Cabanillas, Encargada de Postulaciones y Base de Datos.

- Carolina Botto, Secretaria de Dirección.

- Jenny Cortés, Secretaria del Centro.

- Ricardo Rojas, Auxiliar Administrativo.

\section{6. Área de Desarrollo Institucional}

- Christine Cervenak, Directora del Área.

- Lezak Shallat. Periodista.

\section{ii. Consejo Consultivo Nacional y Comité Asesor Internacional}

\section{Consejo Consultivo Nacional}

Este Consejo está formado por las siguientes personas pertenecientes a la Facultad de Derecho de la Universidad de Chile: Enrique Barros B., Antonio Bascuñán V., Alfredo Etcheberry O., María Angélica Figueroa Q., Ximena Fuentes T., María Teresa Infante C., Roberto Nahum A., Pablo Ruiz-Tagle V., y Paulina Veloso V. Asimismo, participan José Luis Cea E., de la Pontificia Universidad Católica de Chile; Elizabeth Lira K., Centro de Ética Universidad Alberto Hurtado; Jorge Mera F., Universidad Diego Portales; Sonia Montecino A., Facultad de Ciencias Sociales Universidad de Chile, y Agustín Squella N., de la Facultad de Derecho Universidad de Valparaíso.

\section{Comité Asesor Internacional}

Conformado por las siguientes personas: Alex Boraine, International Center for Transitional Justice New York; Antônio Cançado Trindade, Facultade de Direito Universidade de Brasilia; Rebecca Cook, University of Toronto Faculty of Law; Ronald Dworkin, New York University School of Law; Elizabeth Evatt, Tribunal Administrativo Banco Mundial; Héctor Faúndez, Centro de Estudios y de Derechos Humanos Universidad Central de Venezuela; Gustavo Gallón, Comisión Colombiana de Juristas; Claudio Grossman, American University Washington College of Law; Thomas Hammarberg, Olof Palme International Center, Estocolmo; Louis Henkin, Columbia Law School; Sofía Macher, Instituto de Defensa Legal del Perú; Juan Méndez, International Center for Transitional Justice New York; Sonia Picado, Instituto Interamericano de Derechos Humanos; Rodolfo Stavenhagen, El Colegio de México; Henry Steiner, Harvard Law School; Patricia T. de Valdez, Memoria Abierta Argentina; Theo Van Boven, Faculty of Law Universiteit Maastricht. 


\section{iii. Reconocimientos y premios a nuestro staff}

Durante el año 2008, la Co-Directora Cecilia Medina obtuvo el "Premio Ciudadanía 2008", otorgado por la Fundación Ideas por su importante contribución a la defensa de los derechos de las personas y la justicia de género.

La Presidenta de la República nombró al Co-Director José Zalaquett como integrante de la Nueva Comisión Bicentenario del Gobierno de Chile, encargada de articular, orientar y preparar los proyectos que conduzcan a que Chile celebre sus 200 años republicanos.

El Director del Programa de Estado de Derecho, Claudio Nash Rojas, obtuvo el grado de Doctor en Derecho, siendo el primer graduado de este programa de estudios de la Facultad de Derecho de la Universidad de Chile. Asimismo, obtuvo la Medalla Doctoral Universidad de Chile.

\section{INFORMACIÓN INSTITUCIONAL}

\section{i. Estadísticas}

Entre el 2002 y 2008, se han realizado los siguientes avances:

\section{a. Staff}

El CDH está compuesto por 19 personas, 5 de ellas extranjeras, 10 ocupan cargos académicos y 9 cargos de administración.

\section{b. Diplomas Internacionales de postítulo}

- 11 cursos semestrales (2002 a 2008).

- 2 cursos in situ en México y Argentina.

- 317 profesionales seleccionados de 1.590 postulaciones.

- Estudiantes provenientes de 19 países de América.

- 630 pasajes aéreos para alumnos (dos veces por curso), profesores y conferencias internacionales.

- 5.000 noches de alojamiento y viático.

- 160 participaciones aproximadas de profesores invitados (un 40\% de ellos extranjeros).

\section{c. Docencia}

- 1.350 alumnos de pregrado (aproximadamente) en Curso Optativo.

- 114 alumnos de talleres de pregrado.

- 25 módulos de clases en cursos de postgrado y múltiples diplomados impartidos por staff académico.

- Participación del personal académico en aproximadamente 48 conferencias internacionales.

- Participación del personal académico en numerosos diplomados y cursos de magíster ofrecidos por la Facultad de Derecho de la Universidad de Chile. 


\section{d. Publicaciones}

- 18 libros.

- 4 libros en preparación.

- 13 compendios de materiales de cursos (actualizados anualmente).

- 6.600 despachos de libros.

- 1.350 páginas de traducciones del inglés al castellano.

\section{e. Eventos}

- Conferencias internacionales en Santiago organizadas por el CDH.

- 6 procesos de preparación y selección de alumnos para participaciones en Concurso de la American University.

\section{f. Sitio Web}

- 10.682 visitas (2003).

- 18.004 visitas (2004).

- 24.273 visitas (2005).

- 35.860 visitas (2006).

- 41.253 visitas (2007).

- 94.420 visitas (2008).

\section{ii. Evaluación de impacto}

Un aspecto prioritario del CDH para el trienio 2008-2010 es la elaboración de informes de impacto a nivel general y por Programas, con el objeto de reflejar y mejorar nuestras actividades, transparentar nuestro trabajo e informar con claridad a nuestras instituciones donantes.

En el curso del 2008 esta tarea se realizó en el Programa Mujeres y Derechos Humanos, específicamente en el Diplomado Derechos Humanos y Mujeres: Teoría y Práctica. Algunas conclusiones de esta evaluación son las siguientes:

- Este Diploma de Postítulo cuenta con más de 100 personas capacitadas entre el 2004 y 2007.

- Los estudiantes permanentemente encuentran maneras de traducir los conocimientos teóricos adquiridos en el Diploma en avances concretos en derechos humanos, demostrando el impacto del $\mathrm{CDH}$ en el trabajo cotidiano a largo plazo en materia de derechos humanos en la región. Este impacto se puede observar en las siguientes áreas: decisiones jurisdiccionales a nivel local; decisiones en el sistema interamericano de protección de los derechos humanos; impacto en legislación y políticas públicas; e influencia a nivel de educación superior y capacitación en general.

- Los estudiantes, generalmente, logran implementar en la práctica sus trabajos finales, a pesar de las numerosas barreras que suelen encontrar a nivel nacional. Asimismo, dirigen su acción a un público diverso, procurando un impacto concreto en la realidad y un desarrollo a largo plazo. 
Las y los participantes del Diploma se organizan y trabajan en conjunto luego de la graduación, lo que se ha visto facilitado por el soporte web de los diplomados.

\section{iii. Instituciones donantes 2008}

Durante el año 2008, el CDH obtuvo el apoyo financiero de los siguientes donantes: Fundación Ford; Gobierno de Chile; Ministerio de Relaciones Exteriores de Noruega; The Foundation Open Society Institute; The Sigrid Rausing Trust; The John Merck Fund; The Overbrook Foundation; The Tinker Foundation, Inc.; UNIFEM (Fondo de Desarrollo de las Naciones Unidas para la Mujer); Embajada de Suiza en Chile; Oficina Regional para América Latina y el Caribe del Alto Comisionado de Naciones Unidas para los Derechos Humanos.

En el marco de la actividad de Fundraising del CDH, el Co-Director, José Zalaquett realizó viajes a Estados Unidos y Canadá para reuniones con organizaciones de cooperación. Asimismo, en noviembre de 2008, viajó a Noruega y Finlandia con el mismo objetivo, dictando además, diversas charlas. 
